\title{
An Approach for Solving Fuzzy Multi-Objective Linear Fractional Programming Problems
}

\author{
Farhana Akond Pramy \\ Department of Mathematics \\ Bangladesh University of Engineering and Technology, Dhaka-1000, Bangladesh \\ E-mail: farhanaakond@ math.buet.ac.bd
}

(Received November 9, 2017; Accepted December 23, 2017)

\begin{abstract}
In this paper, an attempt has been taken to develop a method for solving fuzzy multi-objective linear fractional programming (FMOLFP) problem. Here, at first the FMOLFP problem is converted into (crisp) multi-objective linear fractional programming (MOLFP) problem using the graded mean integration representation (GMIR) method proposed by Chen and Hsieh. That is, all the fuzzy parameters of FMOLFP problem are converted into crisp values. Then the MOLFP problem is transformed into a single objective linear programming (LP) problem using a proposal given by Nuran Guzel. Finally the single objective LP problem is solved by regular simplex method which yields an efficient solution of the original FMOLFP problem. To show the efficiency of our proposed method, three numerical examples are illustrated and also for each example, a comparison is drawn between our proposed method and the respected existing method.
\end{abstract}

Keywords- Fuzzy set, Linear fractional programming (LFP), Multi-objective linear fractional programming (MOLFP), Fuzzy multi-objective linear fractional programming (FMOLFP) and Graded mean integration representation (GMIR).

\section{Introduction}

Making decisions is an essential part of our daily lives. Almost all decision problems we deal with have multiple, usually conflicting, criteria. Multi-objective programming is such kind of multiple criteria decision making that involves mathematical optimization problems where more than one objective function have to be optimized simultaneously. To deal with many decisionmaking problems in real life, evaluation of fuzzy data is needed. The fuzzy set theory is helpful to deal with the imprecision and vagueness in the measurement of a given quantity.

Fractional programming involves the optimization of one or several ratios of functions subject to some linear restrictions. In literature, various methods can be observed to solve different models of linear fractional programming (LFP) problem. Among the solution methods, the transformation technique developed by Charnes and Cooper (1962), the simplex based algorithm proposed by Swarup (1965) are widely accepted. The simplex method for solving LFP problem described by Bajaliov (2003) is similar to Swarup's method.

When one or more objectives of a multi-objective programming problem are the linear fractional i.e. ratio of two linear functions under some technological linear restrictions, then the problem is known as "multi-objective linear fractional programming (MOLFP) problem".

When there is more than one fractional objective function, it is tough to find the optimal solutions of these problems. When several fractional objective functions are present, the optimal solution for an objective function may not be an optimal solution for some other objective functions. Therefore, one needs to use the concept of the "best compromise solution", also known as "non- 
International Journal of Mathematical, Engineering and Management Sciences

Vol. 3, No. 3, 280-293, 2018

https://dx.doi.org/10.33889/IJMEMS.2018.3.3-020

dominated solution", "efficient solution", "Pareto optimal solution", "Pareto efficient solution" etc. Thus, in MOLFP problem, the notion of efficiency is introduced to replace with that of optimality. A solution is called efficient if none of the objective functions can be developed in value without demeaning the value of any other objective.

Very often in real life situations, the values of coefficients of multi-objective linear fractional programming problems are only imprecisely available to the expert. To handle this type of situation, it would be preferable to interpret the coefficients as fuzzy numerical data. Bellman and Zadeh (1970) first introduced the concept of fuzzy decision making.

Again, to make a comparison among fuzzy alternatives, it is useful to convert fuzzy numbers into crisp numbers. Chen and Hsieh (1999) worked together and came up with graded mean integration representation method to represent generalized fuzzy numbers.

To solve MOLFP problem, few approaches have been reported in the early age. Kornbluth and Steuer (1981) proposed a simplex type algorithm for finding all weakly efficient vertices of the augmented feasible region. Benson (1985) observed that the procedure of Kornbluth and Steuer may not work all the time to compute the numbers for finding break points. Then he presented a fail-safe method to compute these numbers.

In the modern age, some new approaches have also been reported to solve MOLFP and FMOLFP problems. Jain (2012) proposed a method using Gauss elimination technique to derive numerical solution of multi-objective linear programming (MOLP) problem. Then Jain (2014) extended his work for MOLFP problem. Porchelvi et al. (2014) presented procedures for solving both MOLFP problem and FMOLFP problem using the complementary development method of Dheyab (2012), where the fractional linear programming is transformed into a linear programming problem. Guzel and Sivri (2005) presented a method for finding an efficient solution of MOLFP problem using goal programming. Later Guzel (2013) proposed a simplex type algorithm for finding an efficient solution of MOLFP problem based on a theorem studied in a work by Dinkelbach (1967), where he converted the main problem into a single objective LP problem. Moumita and De (2014) presented a method to find a pareto optimal solution of FMOLFP Problem. Muruganandam and Ambika (2017) proposed a technique using harmonic mean to solve FMOLFP Problem.

In this paper, we have developed a method for solving FMOLFP problem by combining the methods proposed by Chen and Hsieh (1999) and Guzel (2013).

\section{Preliminaries}

\subsection{Fuzzy Set}

A fuzzy set $\tilde{A}$ in $X$ is a set of ordered pairs:

$\tilde{A}=\left\{\left(x, \mu_{\tilde{A}}(x)\right): x \in \mathrm{X}\right\}$.

Here $\mu_{\tilde{A}}(x)$ is interpreted as the membership function and the values of $\mu_{\tilde{A}}(x)$ at $x$ represent the "grade of membership" of $x \in \mathrm{X}$ in $\tilde{A}$. The membership function $\mu_{\tilde{A}}(x)$ associates with each point $x \in \mathrm{X}$ a real number in the interval $[0,1]$. 
International Journal of Mathematical, Engineering and Management Sciences

Vol. 3, No. 3, 280-293, 2018

https://dx.doi.org/10.33889/IJMEMS.2018.3.3-020

\subsection{Fuzzy Number}

A fuzzy number $\tilde{A}$ is a fuzzy set defined on $\mathbb{R}$ (membership function $\mu_{\tilde{A}}: \mathbb{R} \rightarrow[0,1]$ ), whose membership function satisfies the following properties

(i) $\tilde{A}$ is normal i.e. there exist an element $x_{0} \in \mathbb{R}$ such that $\mu_{\tilde{A}}\left(x_{0}\right)=1$.

(ii) $\tilde{A}$ is convex i.e. $\left.\mu_{\tilde{A}}\left[\lambda x_{1}+(1-\lambda) x_{2}\right)\right] \geq \min \left\{\mu_{\tilde{A}}\left(x_{1}\right), \mu_{\tilde{A}}\left(x_{2}\right)\right\} ; x_{1}, x_{2} \in \mathbb{R}, \forall \lambda \in[0,1]$.

(iii) $\mu_{\tilde{A}}$ is upper continuous.

(iv) $\operatorname{supp}(\tilde{A})$ is bounded, where $\operatorname{supp}(\tilde{A})=\left\{x \in \mathrm{X}: \mu_{\tilde{A}}(x)>0\right\}$.

\subsection{Generalized Fuzzy Number}

A generalized fuzzy number $\tilde{A}$ is a fuzzy set defined on $\mathbb{R}$ whose membership function satisfies the following properties

(i) $\mu_{\tilde{A}}(x)$ is a continuous mapping from $\mathbb{R}$ to $[0,1]$.

(ii) $\mu_{\tilde{A}}(x)=0,-\infty<x \leq a$.

(iii) $\mu_{\tilde{A}}(x)$ is strictly increasing on $[a, b]$.

(iv) $\mu_{\tilde{A}}(x)=1, b \leq x \leq c$.

(v) $\mu_{\tilde{A}}(x)$ is strictly decreasing on $[c, d]$.

(vi) $\mu_{\tilde{A}}(x)=0, d \leq x<\infty$.

where $a, b, c, d$ are real numbers.

\subsection{Trapezoidal Fuzzy Number}

A fuzzy set $\widetilde{\mathrm{A}}$ defined on $\mathbb{R}$ is called trapezoidal fuzzy number and is denoted by $\widetilde{\mathrm{A}}=(a, b, c, d)$ if the membership function of $\widetilde{\mathrm{A}} i s$ given by,

$$
\mu_{\tilde{A}}(x)=\left\{\begin{array}{cl}
\frac{\mathrm{a}-x}{\mathrm{a}-\mathrm{b}}, & \text { if } \mathrm{a} \leq x \leq \mathrm{b} \\
1, & \text { if } \mathrm{b}<x<c \\
\frac{\mathrm{d}-x}{\mathrm{~d}-\mathrm{c}} & , \text { if } \mathrm{c} \leq x \leq \mathrm{d} \\
0, & \text { otherwise }
\end{array} ; a \leq b \leq c \leq d .\right.
$$

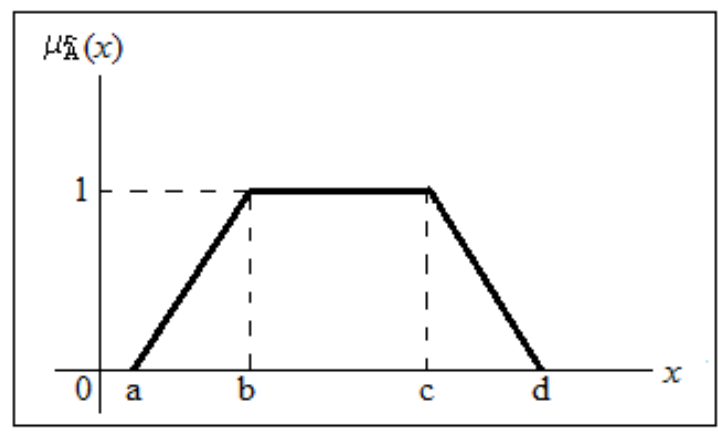

Fig. 1. Trapezoidal fuzzy number

Note: If $b$ and $c$ are equal, then the trapezoidal fuzzy number becomes a triangular fuzzy number as shown in Fig. 2 and is denoted as $(a, b, d)$. 
International Journal of Mathematical, Engineering and Management Sciences

Vol. 3, No. 3, 280-293, 2018

https://dx.doi.org/10.33889/IJMEMS.2018.3.3-020

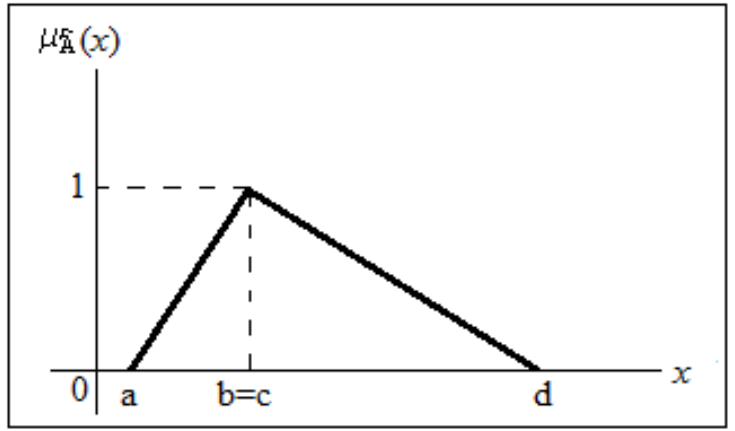

Fig. 2. Triangular fuzzy number

\subsection{Defuzzification}

Defuzzification is a procedure of transforming fuzzy values to crisp values. Defuzzification method provides a correspondence from the set of all fuzzy sets into the set of all real numbers.

\subsection{Multi-Objective Linear Fractional Programming (MOLFP) Problem}

An MOLFP problem is defined as follows

(MOLFP) Maximize $\left\{\boldsymbol{Z}(\boldsymbol{x})=\left(z_{1}(\boldsymbol{x}), z_{2}(\boldsymbol{x}), \ldots, z_{k}(\boldsymbol{x})\right)\right\}$

subject to $A \boldsymbol{x} \leqq \boldsymbol{b}$

$$
\boldsymbol{x} \geqq \mathbf{0} \text {. }
$$

Here,

$$
\begin{aligned}
& S=\left\{\boldsymbol{x} \in \mathbb{R}^{n} \mid A \boldsymbol{x} \leqq \boldsymbol{b}, \boldsymbol{x} \geqq \mathbf{0}, \boldsymbol{b} \in \mathbb{R}^{m}\right\} \text { is the feasible set in decision space, } \\
& A \text { is an } m \times n \text { matrix, } \\
& \boldsymbol{x} \in \mathbb{R}^{n} \text { and } \boldsymbol{b} \in \mathbb{R}^{m} ;(\boldsymbol{b} \geqq \mathbf{0}), \\
& k \geq 2, \\
& z_{i}(\boldsymbol{x})=\frac{\boldsymbol{c}_{i}^{T} \boldsymbol{x}+\alpha_{i}}{\boldsymbol{d}_{i}^{T} \boldsymbol{x}+\beta_{i}}=\frac{N_{i}(\boldsymbol{x})}{D_{i}(\boldsymbol{x})} ; \boldsymbol{c}_{i}^{T}, \boldsymbol{d}_{i}^{T} \in \mathbb{R}^{n} ; \alpha_{i}, \beta_{i} \in \mathbb{R} ; \text { for all } i=1,2, \ldots, k \\
& \text { and } D_{i}(\boldsymbol{x})=\boldsymbol{d}_{i}^{T} \boldsymbol{x}+\beta_{i}>0, \text { for all } i=1,2, \ldots, k, \text { for all } \boldsymbol{x} \in S .
\end{aligned}
$$

\subsection{Efficient Solution of MOLFP Problem}

A solution $\overline{\boldsymbol{x}} \in S$ is an efficient solution of the problem (MOLFP) if and only if there is no $\boldsymbol{x} \in S$ such that $z_{i}(\boldsymbol{x}) \geq z_{i}(\overline{\boldsymbol{x}})$ for all $i=1,2, \ldots, k$ and $z_{i}(\boldsymbol{x})>z_{i}(\overline{\boldsymbol{x}})$ for at least one $\mathrm{i}$.

Note that, for vectors and $\boldsymbol{y}$;

$\boldsymbol{x} \geqq \boldsymbol{y}$ implies $x_{i} \geq y_{i}$ for each $i$,

$\boldsymbol{x} \geq \boldsymbol{y}$ implies $x_{i} \geq y_{i}$ for $i$ and $x_{r}>y_{r}$ for at least one $i=r$

and $\boldsymbol{x}>\boldsymbol{y}$ implies $x_{i}>y_{i}$ for each $i$.

\subsection{Fuzzy Multi-Objective Linear Fractional Programming Problem}

If one or more parameters in the objective functions or mathematical constraints of MOLFP problem are fuzzy numbers, then the problem is called FMOLFP problem. Variables in FMOLFP problem can also be fuzzy numbers. In case of all the parameters and variables being fuzzy numbers, the problem is known as fully FMOLFP problem. 
International Journal of Mathematical, Engineering and Management Sciences

Vol. 3, No. 3, 280-293, 2018

https://dx.doi.org/10.33889/IJMEMS.2018.3.3-020

Let us consider the following form of FMOLFP problem

(FMOLFP) $\quad$ Maximize $\left\{\widetilde{\boldsymbol{Z}}(\boldsymbol{x})=\left(\tilde{z}_{1}(\boldsymbol{x}), \tilde{z}_{2}(\boldsymbol{x}), \ldots, \tilde{z}_{k}(\boldsymbol{x})\right)\right\}$

subject to $\tilde{A} \boldsymbol{x} \leqq \widetilde{\boldsymbol{b}}$

$\boldsymbol{x} \geqq \mathbf{0}$.

Here,

$S=\left\{\boldsymbol{x} \in \mathbb{R}^{n} \mid \tilde{A} \boldsymbol{x} \leqq \widetilde{\boldsymbol{b}}, \boldsymbol{x} \geqq \mathbf{0}\right\}$ is the feasible set in decision space,

$\tilde{A}$ is an $m \times n$ fuzzy matrix,

$\boldsymbol{x} \in \mathbb{R}^{n}$ and $\widetilde{\boldsymbol{b}}$ is an $\mathrm{m}$ dimensional fuzzy vector,

$k \geq 2$,

$\tilde{z}_{i}(\boldsymbol{x})=\frac{\tilde{\boldsymbol{c}}_{i}^{T} \boldsymbol{x}+\widetilde{\alpha}_{i}}{\widetilde{\boldsymbol{d}}_{i}^{T} \boldsymbol{x}+\widetilde{\beta}_{i}} ; \tilde{\boldsymbol{c}}_{i}^{T}, \widetilde{\boldsymbol{d}}_{i}^{T}$ are n dimensional fuzzy vectors; $\tilde{\alpha}_{i}, \tilde{\beta}_{i}$ are fuzzy scalars;

$$
\text { for all } i=1,2, \ldots, k
$$

and $D_{i}(\boldsymbol{x})=\widetilde{\boldsymbol{d}}_{i}^{T} \boldsymbol{x}+\beta_{i}>0$, for all $i=1,2, \ldots, k$, for all $\boldsymbol{x} \in S$.

In this paper, the fuzzy numbers under consideration are triangular or trapezoidal fuzzy numbers.

\section{Graded Mean Integration Representation Method}

Chen and Hsieh (1999) introduced Graded Mean Integration Representation Method for the defuzzifying generalized fuzzy number. The method is basically based on the integral value of graded mean h-level of the generalized fuzzy number.

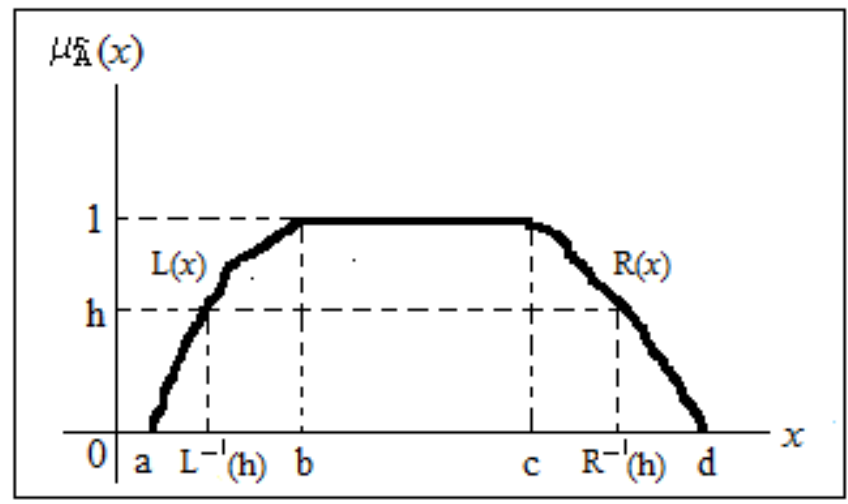

Fig. 3. The graded mean h-level value of generalized fuzzy number $\widetilde{A}$

Let $\widetilde{\mathrm{A}}=(a, b, c, d)_{L R}$ be a generalized fuzzy number with left reference number $\mathrm{L}$ and right reference number $\mathrm{R}$. And $\mathrm{L}^{-1}$ and $\mathrm{R}^{-1}$ be the inverse functions of $\mathrm{L}$ and $\mathrm{R}$ respectively and the graded mean h-level value of the generalized fuzzy number $\widetilde{\mathrm{A}}=(a, b, c, d)_{L R}$ is,

$\frac{\mathrm{h}}{2}\left\{\mathrm{~L}^{-1}(\mathrm{~h})+\mathrm{R}^{-1}(\mathrm{~h})\right\}$. 
International Journal of Mathematical, Engineering and Management Sciences

Vol. 3, No. 3, 280-293, 2018

https://dx.doi.org/10.33889/IJMEMS.2018.3.3-020

The graded mean integration representation of $\widetilde{\mathrm{A}}$ is,

$F(\widetilde{\mathrm{A}})=\frac{\int_{0}^{1} \frac{h}{2}\left\{L^{-1}(h)+R^{-1}(h)\right\} d h}{\int_{0}^{1} h d h} ; 0<h \leq 1$.

Using this formula, the graded mean integration representation of trapezoidal fuzzy number $\widetilde{\mathrm{A}}=$ $(a, b, c, d)$ and triangular fuzzy number $\widetilde{\mathrm{B}}=(a, b, d)$ are given by, respectively,

$F(\widetilde{\mathrm{A}})=\frac{a+2 b+2 c+d}{6}$

$F(\widetilde{\mathrm{B}})=\frac{a+4 b+d}{6}$

\section{A Method for Converting MOLFP Problem into LP Problem}

Here we are going to discuss the method proposed by Guzel (2013) for converting MOLFP problem into LP problem. The method is mainly based on a theorem proposed by Dinkelbach (1967) which is given by,

Theorem 1: $z^{*}=\frac{N\left(x^{*}\right)}{D\left(x^{*}\right)}=\max \left\{\frac{N(x)}{D(x)} \mid x \in S\right\}$ if and only if

$\mathrm{f}\left(z^{*}\right)=\mathrm{f}\left(z^{*}, \boldsymbol{x}^{*}\right)=\max \left\{N(\boldsymbol{x})-z^{*} D(\boldsymbol{x}) \mid \boldsymbol{x} \in S\right\}=0$.

Guzel proposed that, $\overline{\boldsymbol{x}}$ is an efficient solution of the problem (MOLFP) if $\overline{\boldsymbol{x}}$ is an optimal solution of the problem

$\max \left\{\sum_{i=1}^{k}\left(N_{i}(\boldsymbol{x})-z_{i}^{*} D_{i}(\boldsymbol{x})\right) \mid \boldsymbol{x} \in S\right\}$,

where $z_{i}^{*}=\frac{N_{i}\left(x_{i}^{*}\right)}{D_{i}\left(x_{i}^{*}\right)}=\max \left\{\frac{N_{i}(\boldsymbol{x})}{D_{i}(\boldsymbol{x})} \mid x \in S\right\}$ for all $i=1,2, \ldots, k$.

So, MOLFP problem is reduced to an LP problem. Now, let us describe the logic behind this proposal.

Each objective function $z_{i}(\boldsymbol{x})$ of an MOLFP problem can be maximized subject to the given set of constraints using any of the methods proposed by Charnes and Cooper (1962) or Swarup (1965) or Bajaliov (2003) for solving LFP problem.

Let $\boldsymbol{x}_{i}^{*}$ and $z_{i}^{*}$ be the global maximum points and values of each objective function of (MOLFP) respectively, that is,

$z_{i}^{*}=z_{i}\left(\boldsymbol{x}_{i}^{*}\right)=\frac{N_{i}\left(x_{i}^{*}\right)}{D_{i}\left(\boldsymbol{x}_{i}^{*}\right)}=\max \left\{\frac{N_{i}(\boldsymbol{x})}{D_{i}(\boldsymbol{x})} \mid \boldsymbol{x} \in S\right\}$ for all $i=1,2, \ldots, k$ 
International Journal of Mathematical, Engineering and Management Sciences

Vol. 3, No. 3, 280-293, 2018

https://dx.doi.org/10.33889/IJMEMS.2018.3.3-020

Thus, we can write,

$\frac{N_{i}\left(\boldsymbol{x}_{i}^{*}\right)}{D_{i}\left(\boldsymbol{x}_{i}^{*}\right)} \geq \frac{N_{i}(\boldsymbol{x})}{D_{i}(\boldsymbol{x})}$

or $D_{i}(\boldsymbol{x}) \frac{N_{i}\left(\boldsymbol{x}_{i}^{*}\right)}{D_{i}\left(\boldsymbol{x}_{i}^{*}\right)} \geq N_{i}(\boldsymbol{x})$

or $N_{i}(\boldsymbol{x})-z_{i}^{*} D_{i}(\boldsymbol{x}) \leq 0$ for all $i=1,2, \ldots, k$ and for all $\boldsymbol{x} \in S$

Again, let $\overline{\boldsymbol{x}}$ be an optimal solution of the problem:

$\max \left\{\sum_{i=1}^{k}\left(N_{i}(\boldsymbol{x})-z_{i}^{*} D_{i}(\boldsymbol{x})\right) \mid \boldsymbol{x} \in S\right\}$ or $\max \left\{\sum_{i=1}^{k}\left(N_{i}(\boldsymbol{x})-\frac{N_{i}\left(\boldsymbol{x}_{i}^{*}\right)}{D_{i}\left(\boldsymbol{x}_{i}^{*}\right)} D_{i}(\boldsymbol{x})\right) \mid \boldsymbol{x} \in S\right\}$.

Therefore, we have,

$\sum_{i=1}^{k}\left(N_{i}(\boldsymbol{x})-z_{i}^{*} D_{i}(\boldsymbol{x})\right) \leq \sum_{i=1}^{k}\left(N_{i}(\overline{\boldsymbol{x}})-z_{i}^{*} D_{i}(\overline{\boldsymbol{x}})\right) \leq \sum_{\leq 0}^{k} \max _{i=1}\left\{N_{i}(\boldsymbol{x})-z_{i}^{*} D_{i}(\boldsymbol{x})\right\}$

From these inequalities we obtain,

$N_{i}(\boldsymbol{x})-z_{i}^{*} D_{i}(\boldsymbol{x}) \leq N_{i}(\overline{\boldsymbol{x}})-z_{i}^{*} D_{i}(\overline{\boldsymbol{x}}) \leq 0$

$\operatorname{or}\left[\frac{N_{i}(\boldsymbol{x})}{D_{i}(\boldsymbol{x})}-z_{i}^{*}\right] D_{i}(\boldsymbol{x}) \leq\left[\frac{N_{i}(\overline{\boldsymbol{x}})}{D_{i}(\overline{\boldsymbol{x}})}-z_{i}^{*}\right] D_{i}(\overline{\boldsymbol{x}})$

or $\left[\frac{N_{i}(\boldsymbol{x})}{D_{i}(\boldsymbol{x})}-z_{i}^{*}\right] \leq \frac{D_{i}(\overline{\boldsymbol{x}})}{D_{i}(\boldsymbol{x})}\left[\frac{N_{i}(\overline{\boldsymbol{x}})}{D_{i}(\overline{\boldsymbol{x}})}-z_{i}^{*}\right]$

Using theorem 1 , we have both $\left[\frac{N_{i}(\boldsymbol{x})}{D_{i}(\boldsymbol{x})}-z_{i}^{*}\right]$ and $\left[\frac{N_{i}(\overline{\boldsymbol{x}})}{D_{i}(\overline{\boldsymbol{x}})}-z_{i}^{*}\right]$ are non-positive.

Thus the inequality $\frac{D_{i}(\overline{\boldsymbol{x}})}{D_{i}(\boldsymbol{x})} \geq 1$ implies,

$\frac{D_{i}(\overline{\boldsymbol{x}})}{D_{i}(\boldsymbol{x})}\left[\frac{N_{i}(\overline{\boldsymbol{x}})}{D_{i}(\overline{\boldsymbol{x}})}-z_{i}^{*}\right] \leq\left[\frac{N_{i}(\overline{\boldsymbol{x}})}{D_{i}(\overline{\boldsymbol{x}})}-z_{i}^{*}\right]$

or $\frac{N_{i}(\boldsymbol{x})}{D_{i}(\boldsymbol{x})}-z_{i}^{*} \leq \frac{N_{i}(\overline{\boldsymbol{x}})}{D_{i}(\overline{\boldsymbol{x}})}-z_{i}^{*}$

[Using (5)]

or $\frac{N_{i}(\boldsymbol{x})}{D_{i}(\boldsymbol{x})} \leq \frac{N_{i}(\overline{\boldsymbol{x}})}{D_{i}(\overline{\boldsymbol{x}})}$

or $z_{i}(\boldsymbol{x}) \leq z_{i}(\overline{\boldsymbol{x}})$ for all $i$. 
International Journal of Mathematical, Engineering and Management Sciences

Vol. 3, No. 3, 280-293, 2018

https://dx.doi.org/10.33889/IJMEMS.2018.3.3-020

Hence we have, $\bar{x}$ is an efficient solution of the problem (MOLFP).

Now, if $\bar{x}$ is not an efficient solution of the problem (MOLFP); then there exists $\boldsymbol{x} \in S$ such that,

$\frac{N_{i}(\bar{x})}{D_{i}(\bar{x})} \leq \frac{N_{i}(x)}{D_{i}(\boldsymbol{x})}$ for all $i$ and $\frac{N_{j}(\bar{x})}{D_{j}(\bar{x})}<\frac{N_{j}(x)}{D_{j}(x)}$ for at least one $j$.

Then using (3) we have,

$\frac{N_{i}(\overline{\boldsymbol{x}})}{D_{i}(\overline{\boldsymbol{x}})} \leq \frac{N_{i}(\boldsymbol{x})}{D_{i}(\boldsymbol{x})} \leq z_{i}^{*}$ or $N_{i}(\overline{\boldsymbol{x}})-z_{i}^{*} D_{i}(\overline{\boldsymbol{x}}) \leq N_{i}(\boldsymbol{x})-z_{i}^{*} D_{i}(\boldsymbol{x})$ for all $i$

and $\frac{N_{j}(\overline{\boldsymbol{x}})}{D_{j}(\overline{\boldsymbol{x}})}<\frac{N_{j}(\boldsymbol{x})}{D_{j}(\boldsymbol{x})}<z_{j}^{*}$ or $N_{j}(\overline{\boldsymbol{x}})-z_{j}^{*} D_{j}(\overline{\boldsymbol{x}})<N_{j}(\boldsymbol{x})-z_{j}^{*} D_{j}(\boldsymbol{x})$ for at least one $j$.

Summing the k inequalities, we get,

$\sum_{i=1}^{k} N_{i}(\overline{\boldsymbol{x}})-z_{i}^{*} D_{i}(\overline{\boldsymbol{x}}) \leq \sum_{i=1}^{k} N_{i}(\boldsymbol{x})-z_{i}^{*} D_{i}(\boldsymbol{x})$

which contradicts that $\bar{x}$ is an optimal solution of the problem:

$\max \left\{\sum_{i=1}^{k}\left(N_{i}(\boldsymbol{x})-z_{i}^{*} D_{i}(\boldsymbol{x})\right) \mid \boldsymbol{x} \in S\right\}$

Hence, the proposal of Guzel is verified.

\section{Our Proposed Approach for Solving Fuzzy Multi-Objective Linear Fractional Programming Problem}

We first defuzzify the FMOLFP problem using the graded mean integration representation method; as a result, the problem converts to MOLFP problem. Then we transform MOLFP problem into an LP problem using Guzel's proposal. Finally, the LP problem is solved by simplex method, whose optimal solution is the required efficient solution of the original problem.

Now, we summarize the working procedure to solve the problem (FMOLFP) step by step as follows

Step I: Convert the FMOLFP problem to an MOLFP problem using GMIR method, i.e. convert each fuzzy number involved in the FMOLFP to crisp number using (1) or (and) (2).

Step II: Find the maximum value $z_{i}^{*}$ of each objective function $z_{i}(\boldsymbol{x})$ in MOLFP problem subject to the (crisp) set of constraints.

Step III: Formulate the equivalent LP problem of the MOLFP problem:

$\max \left\{\sum_{i=1}^{k}\left(N_{i}(\boldsymbol{x})-z_{i}^{*} D_{i}(\boldsymbol{x})\right) \mid \boldsymbol{x} \in S\right\}$; subject to the constraints of MOLFP problem. 
International Journal of Mathematical, Engineering and Management Sciences

Vol. 3, No. 3, 280-293, 2018

https://dx.doi.org/10.33889/IJMEMS.2018.3.3-020

Step IV: Find an optimal solution of the LP problem obtained in step III. This solution is an efficient solution of the MOLFP problem as well as the FMOLFP problem.

\section{Numerical Examples}

Example 1: This example is taken from Moumita and De (2014).

Maximize $\tilde{z}_{1}=\frac{(4,7,10,12) x_{1}+(8,10,14,15) x_{2}+(2.5,4,7.5,11.5) x_{3}+(2,3,4,6)}{(10,14,20,22) x_{1}+(20,23.5,27.5,29) x_{2}+(18,20,25,28) x_{3}+(5,10,18,20)}$

Maximize $\tilde{z}_{2}=\frac{(20,24,28) x_{1}+(18,25,30) x_{2}+(14,19,25) x_{3}+(1,6,10)}{(14,16,19,23) x_{1}+(18,21,25,27) x_{2}+(15,20,25,30) x_{3}+(10,15,20,25)}$

Subject to

$(10,17,19,25) x_{1}+(14,16,22,24) x_{2}+(20,25,27,30) x_{3} \leq 44.96$

$(0.03,0.07,0.09) x_{1}+(0.05,0.08,0.1) x_{2}+(0.02,0.06,0.07) x_{3} \leq 0.9163$

$(4,6,10,13) x_{1}+(0,5,10,15) x_{2}+(8,11,14,20) x_{3} \leq 35$

$x_{1}, x_{2}, x_{3} \geq 0$.

Solution: Using GMIR method we convert the given FMOLFP problem into the following MOLFP problem:

Maximize $z_{1}=\frac{8.3 x_{1}+11.8 x_{2}+6.2 x_{3}+3.7}{\frac{16.7 x_{1}+25.2 x_{2}+22.7 x_{3}+13.5}{24 x_{1}+24.7 x_{2}+19.2 x_{3}+5.8}}$

Maximize $z_{2}=\frac{24 x_{1}+24.7 x_{2}+19.2 x_{3}+5.8}{17.8 x_{1}+22.8 x_{2}+22.5 x_{3}+17.5}$

Using GMIR method,
$(4,7,10,12)=\frac{4+2 \times 7+2 \times 10+12}{6}=8.3$
$(20,24,28)=\frac{20+4 \times 24+28}{6}=24$
$(10,17,19,25)=\frac{10+2 \times 17+2 \times 19+25}{6}=17.8$
and so on.

Subject to

$17.8 x_{1}+19 x_{2}+25.7 x_{3} \leq 44.96$

$0.07 x_{1}+0.06 x_{2}+0.06 x_{3} \leq 0.9163$

$8.2 x_{1}+7.5 x_{2}+13 x_{3} \leq 35$

$x_{1}, x_{2}, x_{3} \geq 0$.

To solve this MOLFP problem, we find the optimal value of each of the objective functions $z_{1}(\boldsymbol{x}), z_{2}(\boldsymbol{x})$ subject to the above constraints, using any of the methods for solving LFP problems (for convenience we use MATHEMATICA). We get,

$\max z_{1}=0.442956=z_{1}^{*}$ at $\left(2.52584,1.45878 \times 10^{-9}, 0\right)$,

$\max z_{2}=1.0634=z_{2}^{*}$ at $(2.52584,0,0)$.

An LP problem, which is equivalent to the MOLFP problem, is constructed according to the proposed algorithm as follows:

$$
\begin{aligned}
\max z(\boldsymbol{x})=( & \left.8.3 x_{1}+11.8 x_{2}+6.2 x_{3}+3.7\right)-z_{1}^{*}\left(16.7 x_{1}+25.2 x_{2}+22.7 x_{3}+13.5\right) \\
& +\left(24 x_{1}+24.7 x_{2}+19.2 x_{3}+5.8\right)-z_{2}^{*}\left(17.8 x_{1}+22.8 x_{2}+22.5 x_{3}+17.5\right) \\
& =5.974 x_{1}+1.092 x_{2}-8.582 x_{3}-15.089
\end{aligned}
$$


International Journal of Mathematical, Engineering and Management Sciences

Vol. 3, No. 3, 280-293, 2018

https://dx.doi.org/10.33889/IJMEMS.2018.3.3-020

Subject to

$17.8 x_{1}+19 x_{2}+25.7 x_{3} \leq 44.96$

$0.07 x_{1}+0.06 x_{2}+0.06 x_{3} \leq 0.9163$

$8.2 x_{1}+7.5 x_{2}+13 x_{3} \leq 35$

$x_{1}, x_{2}, x_{3} \geq 0$.

Solving this LP problem by regular simplex method (for convenience we use Mathematica), we get the following optimal solution

$x_{1}=2.52584, x_{2}=0, x_{3}=0$.

Hence, an efficient solution of the FMOLFP problem is

$x_{1}=2.52584, x_{2}=0, x_{3}=0$ with $\max z_{1}=0.442956, \max z_{2}=1.0634$.

Comparison: The method proposed by Moumita and De (2014) gives the following result

$\max z_{1}=0.43, \max z_{2}=0.92$.

So, comparing the maximum values of $z_{1}, z_{2}$; our method gives quite better result.

Example 2: This example is taken from Muruganandam and Ambika (2017).

Maximize $\tilde{z}_{1}=\frac{(2,4,6,8) x_{1}+(5,7,9,11) x_{2}}{(0,1,3,4) x_{1}+(0,1,1,2) x_{2}-(1,1,1,1)}$

Maximize $\tilde{z}_{2}=\frac{(4,7,9,12) x_{1}+(3,4,6,7) x_{2}}{(2,3,5,6) x_{1}+(0,1,3,4) x_{2}-(1,2,2,3)}$

Maximize $\tilde{z}_{3}=\frac{(0,1,3,4) x_{1}-(5,9,11,15) x_{2}}{(3,5,7,9) x_{1}+(1,2,4,5) x_{2}-(0,1,5,6)}$

Maximize $\tilde{z}_{4}=\frac{-(1,3,5,7) x_{1}-(2,5,7,10) x_{2}}{(-1,1,3,5) x_{1}+(1,1,1,1) x_{2}-(0,1,1,2)}$

Subject to

$(0,1,3,4) x_{1}+(1,3,7,9) x_{2} \geq(6,8,12,14)$

$(0,3,5,8) x_{1}+(-1,2,4,7) x_{2} \leq(12,18,22,28)$

$-(1,1,1,1) x_{1}+(0,1,1,2) x_{2} \leq(0,1,3,4)$

$(1,1,1,1) x_{1} \leq(-1,1,3,5)$

$(-1,1,1,3) x_{2} \geq(1,2,6,7)$

$x_{1}, x_{2} \geq 0$.

Solution: Using GMIR method we convert the given FMOLFP problem into the following MOLFP problem:

Maximize $z_{1}=\frac{5 x_{1}+8 x_{2}}{2 x_{1}+x_{2}-1}$

Maximize $z_{2}=\frac{8 x_{1}+5 x_{2}}{4 x_{1}+2 x_{2}-2}$ 
International Journal of Mathematical, Engineering and Management Sciences

Vol. 3, No. 3, 280-293, 2018

https://dx.doi.org/10.33889/IJMEMS.2018.3.3-020

Maximize $z_{3}=\frac{2 x_{1}-10 x_{2}}{6 x_{1}+3 x_{2}-3}$

Maximize $z_{4}=\frac{-4 x_{1}-6 x_{2}}{2 x_{1}+x_{2}-1}$

Subject to

$2 x_{1}+5 x_{2} \geq 10$

$4 x_{1}+3 x_{2} \leq 20$

$-x_{1}+x_{2} \leq 2$

$x_{1} \leq 2, x_{2} \geq 4, x_{1}, x_{2} \geq 0$.

At first, we find the optimal value of each of the objective functions $z_{1}(\boldsymbol{x}), z_{2}(\boldsymbol{x}), z_{3}(\boldsymbol{x}), z_{4}(\boldsymbol{x})$ subject to the above constraints. We get,

$$
\begin{aligned}
& \max z_{1}=6=z_{1}^{*} \text { at }(2,4), \\
& \max z_{2}=2.57143=z_{2}^{*} \text { at }(2,4), \\
& \max z_{3}=-1.71429=z_{3}^{*} \text { at }(2,4), \\
& \max z_{4}=-4.57143=z_{4}^{*} \text { at }(2,4) .
\end{aligned}
$$

An LP problem, which is equivalent to the MOLFP problem, is constructed according to the proposed algorithm as follows:

$$
\begin{aligned}
\max z(\boldsymbol{x})=(5 & \left.x_{1}+8 x_{2}\right)-z_{1}^{*}\left(2 x_{1}+x_{2}-1\right)+\left(8 x_{1}+5 x_{2}\right)-z_{2}^{*}\left(4 x_{1}+2 x_{2}-2\right) \\
& +\left(2 x_{1}-10 x_{2}\right)-z_{3}^{*}\left(6 x_{1}+3 x_{2}-3\right)+\left(-4 x_{1}-6 x_{2}\right)-z_{4}^{*}\left(2 x_{1}+x_{2}-1\right) \\
& =8.143 x_{1}-4.429 x_{2}+1.429
\end{aligned}
$$

Subject to

$2 x_{1}+5 x_{2} \geq 10$

$4 x_{1}+3 x_{2} \leq 20$

$-x_{1}+x_{2} \leq 2$

$x_{1} \leq 2, x_{2} \geq 4, x_{1}, x_{2} \geq 0$.

Solving this LP problem, we get the following optimal solution $x_{1}=2, x_{2}=4$.

Hence an efficient solution of the FMOLFP problem is

$x_{1}=2, x_{2}=4$

with $\max z_{1}=6, \max z_{2}=2.57143, \max z_{3}=-1.71429$ and $\max z_{4}=-4.57143$.

Comparison: The method proposed by Muruganandam and Ambika (2017) gives the following Result

$\max z_{1}=6, \max z_{2}=2.57143, \max z_{3}=-1.71429$ and $\max z_{4}=-4.57143$

which is same as the result obtained by our proposed method. 
International Journal of Mathematical, Engineering and Management Sciences

Vol. 3, No. 3, 280-293, 2018

https://dx.doi.org/10.33889/IJMEMS.2018.3.3-020

Example 3: This example is taken from Porchelvi et al. (2014).

Maximize $\tilde{z}_{1}=\frac{(5,7,2,2) x_{1}+(4,6,2,2) x_{2}}{(1,3,2,2) x_{1}+(6,8,2,2)}$

Maximize $\tilde{z}_{2}=\frac{(1,3,2,2) x_{1}+(2,4,2,2) x_{2}}{(0.75,1.25,0.5,0.5) x_{1}+(0.75,1.25,0.5,0.5) x_{2}+(6,8,2,2)}$

Subject to

$x_{1}+2 x_{2} \leq 3$

$3 x_{1}+2 x_{2} \leq 6$

$x_{1}, x_{2} \geq 0$.

Solution: Using GMIR method we convert the given FMOLFP problem into the following MOLFP problem:

Maximize $z_{1}=\frac{4.2 x_{1}+3.7 x_{2}}{2.2 x_{1}+4.7}$

Maximize $z_{2}=\frac{2.2 x_{1}+2.7 x_{2}}{0.8 x_{1}+0.8 x_{2}+4.7}$

Subject to

$x_{1}+2 x_{2} \leq 3$

$3 x_{1}+2 x_{2} \leq 6$

$x_{1}, x_{2} \geq 0$

The optimal value of each of the objective functions $z_{1}(\boldsymbol{x}), z_{2}(\boldsymbol{x})$ subject to the above constraints is as follows:

$\max z_{1}=1.18085=z_{1}^{*}$ at $(0,1.5)$,

$\max z_{2}=0.819231=z_{2}^{*}$ at $(1.5,0.75)$.

An LP problem, which is equivalent to the MOLFP problem, is constructed according to the proposed algorithm as follows:

$$
\begin{gathered}
\max z(\boldsymbol{x})=\left(4.2 x_{1}+3.7 x_{2}\right)-z_{1}^{*}\left(2.2 x_{1}+4.7\right)+\left(2.2 x_{1}+2.7 x_{2}\right)-z_{2}^{*}\left(0.8 x_{1}+0.8 x_{2}+4.7\right) \\
=3.147 x_{1}+5.745 x_{2}-9.4
\end{gathered}
$$

Subject to

$x_{1}+2 x_{2} \leq 3$

$3 x_{1}+2 x_{2} \leq 6$

$x_{1}, x_{2} \geq 0$.

Solving this LP problem, we get the following optimal solution

$x_{1}=1.5, x_{2}=0.75$.

Hence an efficient solution of the given FMOLFP problem is

$x_{1}=1.5, x_{2}=0.75$ with $\max z_{1}=1.13438, \max z_{2}=0.81923$. 
International Journal of Mathematical, Engineering and Management Sciences

Vol. 3, No. 3, 280-293, 2018

https://dx.doi.org/10.33889/IJMEMS.2018.3.3-020

Comparison: The method proposed by Porchelvi et al. (2014) gives the following result

$\max z_{1}=1.13438, \max z_{2}=0.81923 ;$

this is also same as the result obtained by our proposed method.

\section{Conclusion}

In this work, we have tried to find an efficient solution of fuzzy multi-objective linear fractional programming (FMOLFP) problem. We have defuzzified all the fuzzy parameters of the FMOLFP problem by using the graded mean integration representation method, which yields a (crisp) multi-objective linear fractional programming (MOLFP) problem. Then we converted the MOLFP problem into a single objective linear programming (LP) problem. The optimal solution of the LP problem gives an efficient solution of the MOLFP problem as well as the FMOLFP problem. Finally we have explained our proposed method with numerical examples. We have also shown the comparison with the respected existing method for each example. In future, further works can be done to model real life oriented problems as FMOLFP problems and solve them using our proposed method. Also, potential efforts can be given to develop a methodology for solving fully FMOLFP problems.

\section{References}

Bajalinov, E. B. (2003). Linear-fractional programming: theory, methods, applications and software. Kluwer Academic Publishers, Boston.

Bellman, R. E., \& Zadeh, L.A. (1970). Decision making in a fuzzy environment. Management Science, 17, 141-164.

Benson, H. P. (1985). Finding certain weakly-efficient vertices in multiple objective linear fractional programming. Management Science, 31(2), 240-245.

Charnes, A., \& Cooper, W. W. (1962). Programming with linear fractional functionals. Naval Research Logistics, 9(3-4), 181-186.

Chen, S. H., \& Hsieh, C. H. (1999). Graded mean integration representation of generalized fuzzy number. Journal of Chinese Fuzzy Systems, 5(2), 1-7.

Dheyab, A. N. (2012). Finding the optimal solution for fractional linear programming problems with fuzzy numbers. Journal of Kerbala University, 10(3), 105-110

Dinkelbach, W. (1967). On nonlinear fractional programming. Management Science, 13(7), 492-498.

Guzel, N. (2013, November). A proposal to the solution of multiobjective linear fractional programming problem. Abstract and Applied Analysis (Vol. 2013). Hindawi Publishing Corporation.

Guzel, N., \& Sivri, M. (2005). Proposal of a solution to multi objective linear fractional programming problem. Sigma Journal of Engineering and Natural Sciences, 2, 43-50. 
International Journal of Mathematical, Engineering and Management Sciences

Vol. 3, No. 3, 280-293, 2018

https://dx.doi.org/10.33889/IJMEMS.2018.3.3-020

Jain, S. (2012). Modeling of Gauss elimination technique for multi-objective linear programming problem. Journal of Novel Applied Sciences, 1, 25-29.

Jain, S. (2014). Modeling of Gauss elimination technique for multi-objective fractional programming problem. South Asian Journal of Mathematics, 4(3), 148-153.

Kornbluth, J. S., \& Steuer, R. E. (1981). Multiple objective linear fractional programming. Management Science, 27(9), 1024-1039.

Moumita, D., \& De, P. K. (2014). A note on fuzzy multi-objective linear fractional programming problem. International Journal of Computer Science and Network, 3(6), 568-572.

Muruganandam, S., \& Ambika, P. (2017). Harmonic mean technique to solve multi objective fuzzy linear fractional programming problems. Global Journal of Pure and Applied Mathematics, 13(10), 73217329.

Porchelvi, R. S., Vasanthi L., \& Hepzibah, R. I. (2014). On solving multi objective fractional linear programming problems. International Journal of Current Research, 6(8), 8095-8102.

Swarup, K. (1965). Linear fractional functionals programming. Operations Research, 13(6), 1029-1036. 